\title{
IMPORTÂNCIA DO PROGRAMA EDUCACIONAL DE DOAÇÃO E TRANSPLANTE EM ESCOLAS MÉDICAS
}

\author{
Educational program of organ donation and transplantation at medical school
}

\author{
Clotilde Druck Garcia',3, João Carlos Goldani',3, Jorge Neumann ${ }^{3}$, Roberto Chem ${ }^{2,3}$, Eduardo Chem ${ }^{3}$, \\ Jose de Jesus Camargo 2,3, Fernando Lucchese², José Dario Frota Filho³, Italo Marcon 2,3, Alexandre Marcon³, Ajácio Brandão ${ }^{1,3}$, \\ Antonio Kalil2,3, Santo Pascoal Vitola ${ }^{3}$, Viviane Barros Bittencourt ${ }^{3}$, Sérgio Roberto Haussen ${ }^{1,3}$, Lúcia Elbern ${ }^{4}$, Elizabeth de \\ Carvalho Castro ${ }^{6}$, Adriane P Barboza ${ }^{3}$, Elizete Keitel ${ }^{1,3}$, João Rafael Victor Schmitt ${ }^{1,5}$, Sergio Roithmann ${ }^{1}$, Valter Duro Garcia ${ }^{3,5}$
}

\begin{abstract}
RESUMO
Objetivo: Educar precocemente futuros profissionais de saúde, alunos de Medicina, Nutrição e Biomedicina, visando aumentar seu conhecimento sobre doação e transplantes e qualificá-los para divulgar informações, produzindo uma resposta positiva, reforçando seu compromisso com esse processo, para aumentar o número insuficiente de doadores. Métodos: A fim de fornecer as informações necessárias sobre o processo de doação-transplante, a Universidade Federal de Ciências da Saúde de Porto Alegre, associada ao Departamento de Coordenação Hospitalar de Transplante da Santa Casa de Porto Alegre criou um programa educacional de Doação de Órgãos e Transplante. A cada semestre, 50-90 alunos estão matriculados no curso, que envolve um total de 25 horas. Vários aspectos são abordados, como morte encefálica, processo de doação, aspectos políticos e legais da doação e transplantes de pele, pulmão, medula óssea, coração, pâncreas, fígado e renal. Resultados: Os alunos avaliaram o curso e classificaram como excelente, concluindo que ele contribuiu para sua educação. Sua atitude em relação à doação e transplante de órgãos foi fortemente positiva no final do curso. Conclusão: Um conhecimento mais profundo do processo doação-transplante pelos profissionais de saúde é essencial para agilizar a logística para a doação de órgãos e pode influenciar positivamente a decisão das famílias de potenciais doadores. Este projeto visa educar e estimular os alunos no processo de doação-transplante e pode ser considerado como um projeto-piloto bem sucedido a ser aplicado em outras escolas médicas.
\end{abstract}

Descritores: Educação, Doação de Órgão, Transplante.

Instituições:

1 Departamento de Medicina Interna da Universidade Federal de Ciências da Saúde de Porto Alegre - Porto Alegre / RS

2 Departamento de Cirurgia da Universidade Federal de Ciências da Saúde de Porto Alegre Porto Alegre / RS

${ }^{3}$ Centro de Transplantes Hospital Dom Vicente Scherer da Irmandade Santa Casa Misericórdia de Porto Alegre - Porto Alegre / RS

${ }^{4}$ Via Vida Doação e Transplante - Porto Alegre / RS

5 Central de Transplantes do Rio Grande do Sul - Porto Alegre / RS

${ }^{6}$ Departamento de Ciências Básicas da Saúde da Universidade de Ciências da Saúde de Porto Alegre - Porto Alegre / RS

Correspondência:

Clotilde Druck Garcia

Rua Correa Lima, 1493 - Porto Alegre / RS - CEP: 90859-250 - Brasil

Tel.: (51) 32148734 / Fax: (51) 32330833

E-mail: cdgarcia1@uol.com.br

\section{INTRODUÇÃO}

Enquanto aumenta cada vez mais o número de pacientes que poderiam se beneficiar com o transplante de órgãos em decorrência do desenvolvimento de novas tecnologias e técnicas envolvidas nesse processo, o número de doações permanece insuficiente. ${ }^{1}$ Entre os principais obstáculos estão os problemas logísticos como as dificuldades para o diagnóstico de morte encefálica e na manutenção do potencial doador e as elevadas taxas de negativas familiares, motivadas por diversos fatores, como a não-compreensão do conceito de morte encefálica, a idéia da deformação do corpo após a cirurgia de retirada, o medo de comercialização dos órgãos e o não conhecimento do sistema de distribuição e alocação. ${ }^{2,3}$

\section{OBJETIVO}

Educar acadêmicos da área da saúde sobre o processo doaçãotransplante de órgãos, oferecendo-lhes os conhecimentos básicos com a finalidade de engajá-los nesse processo.

\section{MÉTODOS}

Há três anos foi criada a disciplina eletiva de Doação e Transplante de Órgãos, sem exigência de pré-requisitos, oferecida a todos 
1050 Clotilde Druck Garcia, João Carlos Goldani, Jorge Neumann, Roberto Chem, Eduardo Chem, Jose de Jesus Camargo, Fernando Lucchese, José Dario Frota Filho, Italo Marcon, Alexandre Marcon, Ajácio Brandão, Antonio Kalil, Santo Pascoal Vitola, Viviane Barros Bittencourt, Sérgio Roberto Haussen, Lúcia Elbern,

Elizabeth de Carvalho Castro, Adriane P Barboza, Elizete Keitel, João Rafael Victor Schmitt, Sergio Roithmann, Valter Duro Garcia

acadêmicos da então Fundação Faculdade Federal de Ciências Médicas de Porto Alegre, hoje Universidade Federal de Ciências da Saúde de Porto Alegre (UFCSPA). A disciplina consiste de 25 horas de aulas teóricas e é oferecida semestralmente aos alunos nos primeiros semestres de seus cursos (Medicina, Biomedicina, Nutrição e Psicologia). Todos os aspectos do processo doaçãotransplante são apresentados (Figura 1), como diagnóstico de morte encefálica, aspectos políticos e legais da doação, critérios de alocação e noções básicas dos transplantes dos diferentes órgãos. A disciplina conta com a colaboração dos professores da Universidade, coordenadores hospitalares e médicos da Irmandade Santa Casa de Misericórdia de Porto Alegre, voluntários da ONG Via-Vida, funcionários da Central de Transplantes do RS e pacientes transplantados e em lista de espera.

Figura 1. Programa Teórico da Disciplina Doação e Transplante de Órgãos

Introdução da disciplina
Entrevista com pacientes transplantados
Situação atual e perspectivas dos transplantes
Processo doação-transplante
Indicações dos transplantes
Morte encefálica
Alocação de órgãos e tecidos
Doador Vivo - indicações, ética e cuidados
Entrevista familiar - coordenador hospitalar
Central de transplantes
Imunologia dos transplantes
Banco de olhos e transplante de córneas
Transplante e sociedade
Banco de pele e transplante de pele
Transplante de medula óssea
Transplante de rim
Transplante de rim e pâncreas
Transplante de fígado
Transplante de intestino
Transplante de pulmão
Transplante de coração
Ética e transplante

\section{RESULTADOS}

No período de março de 2006 a julho de 2009 participaram 300 acadêmicos. Os alunos avaliaram a disciplina como excelente, sugerindo que fosse organizada uma nova disciplina eletiva mais avançada. A disciplina de Doação e Transplante II foi criada em março de 2007, exclusiva para estudantes de Medicina. Também oferecida semestralmente, está em seu sexto curso, com participação de 100 estudantes até julho de 2009. Essa disciplina inclui acompanhamento de cirurgias de transplantes e de pacientes no pré e pós-operatório, participação em trabalhos científicos, visitas às diversas instituições ligadas ao processo de doação e transplante de órgãos e atuação junto à comunidade, visando conscientizá-la sobre a doação de órgãos (Figura 2). Alguns desses ex-alunos estão organizando a Liga de Transplantes, tendo apresentado vários trabalhos em congressos, participado de eventos educacionais, feiras de saúde com banca de Doação e Transplante e auxiliado a ONG Via-Vida, além de pretenderem iniciar projeto de educação em prevenção e doação de órgãos em escolas primárias. Cabe destacar que os acadêmicos de Medicina desta Instituição têm obtido especial êxito nas provas de seleção para estagiários da Central de Transplantes do Estado, atuando de maneira satisfatória como plantonistas no processo de doação-alocação de órgãos. Após a disciplina, muitos dos alunos engajados no processo de doação e transplante têm formado opinião crítica em relação ao mesmo, podendo no futuro contribuir para seu aperfeiçoamento.

Figura 2. Programa da Disciplina Doação e Transplante /I

\begin{tabular}{ll}
\hline Estágios e Aulas & Carga horária \\
\hline Introdução à disciplina: Logística do Transplante & 2 horas \\
Preparo do Doador e Receptor & 2 horas \\
Preparo do Receptor Pediátrico & Opcional \\
Cirurgia do Receptor & 4 horas \\
Cirurgia do Doador (Nefrectomia) & 2 horas \\
Acompanhamento pós-transplante; UTI e internação & 2 horas \\
Ambulatório pós-transplante & 2 horas \\
Transplante Renal Pediátrico & 2 horas \\
Imunologia de Transplante & 2 horas \\
Alocação de Rim (Central de Transplantes) & 2 horas \\
Pesquisa em Transplante Renal & 6 horas \\
Feira de Saúde - Banca de Doação e Transplante de Órgãos & 2 horas \\
Apresentação e Discussão dos Trabalhos & 2 horas \\
\hline
\end{tabular}

\section{DISCUSSÃO}

As preocupações e a falta de entendimento sobre o processo de doação de órgãos normalmente não são resolvidas apenas com campanhas públicas de conscientização. Uma postura adequada do profissional de saúde em relação à doação de órgãos pode agilizar o processo doação-transplante através do conhecimento do diagnóstico de morte encefálica e da manutenção do potencial doador, além de influenciar na decisão favorável da família à doação. O aumento do conhecimento dos profissionais de saúde sobre doação e transplante de órgãos e sua capacitação para disseminar essas informações pode resultar num aumento do número de doadores. A educação precoce de estudantes da área de saúde é um fator decisivo nesse processo., ${ }^{4,5}$

\section{CONCLUSÃO}

O conhecimento obtido e a motivação apresentada pelos estudantes que participaram da disciplina de doação e transplante já estão trazendo benefícios à nossa população, através da participação deles em várias atividades. Propomos a instituição de disciplinas eletivas nos moldes dessa nas faculdades da área de saúde de todo o País. 


\section{ABSTRACT}

Purpose: The purpose of our program is to supply basic knowledge on organ donation and transplantation to students of Medicine, Nutrition and Biomedicine, aiming to increase their knowledge on donation and transplantation and to qualify them to spread information, and enhance their commitment to this process to produce a positive response to increase the insufficient amount of donors. Methods: The premature education of students in their careers. In order to supply the necessary information on the donation-transplantation process, a Medical School associated to the Hospital Transplant Coordination Department has created an educational program of Organ Donation and Transplantation. This course aims medical, biomedical and nutrition students. Each semester 50 to 90 students are enrolled in the course, involving a total of 25 hours. Various aspects are discussed, such as brain death, donor management, political and legal aspects of the donation, skin, lung, bone marrow, heart, pancreas, liver and kidney transplantation. Results: Students rated the course as excellent, concluding that it contributed to their education. Their attitude towards organ donation and transplantation was strongly positive at the end of the course. Conclusion: A favorable attitude of the health professionals to organ donation can positively influence the decision of families of potential donors. This project aims to educate and stimulate students in the organ donation-transplantation process and it should be implemented in other Medical Schools.

Keywords: Education, Organ Donation, Transplantation

\section{REFERÊNCIAS}

1. Capaverde FB, Londero GG, Figueiredo FM, Hoelfmann N, Oliveira DM, et al Epidemiology of brain death and donation rate in the state of Rio Grande do Sul, Brazil: analysis between 1988 and 2004. Transplant Proc. 2007;39:346.

2. Barcellos FC, Araujo CL, da Costa JD. Organ donation: a population-based study. Clin Transplant. 2005;19:33
3. Tessmer CS, da Silva AR, Barcellos FC, Araujo CL, da Costa JD, et al. Do people accept brain death as death? A study in Brazil. Prog Transplant. 2007;17:63.

4. Rios A, Ramirez P, del mar Rodriguez M, Martínez-Alarcón L, Lucas D, et al. Benefit of a hospital course about organ donation and transplantation: an evaluation by Spanish hospital transplant personnel. Transplant Proc. 2007;39:1310.

5. Burra P, De Bona M, Canova D, D'Aloiso MC, Germani G, et al: Changing attitude to organ donation and transplantation in university students during the years of medical school in Italy. Transplant Proc. 2005;37:547. 